\title{
Observational Learning for Students Diagnosed with Autism: A Review Paper
}

\author{
Donna Townley-Cochran ${ }^{1} \cdot$ Justin B. Leaf $^{1} \cdot$ Mitchell Taubman $^{1} \cdot$ Ronald Leaf $^{1}$ • \\ John McEachin ${ }^{1} \cdot$ Autism Partnership Foundation
}

Received: 5 December 2014 / Accepted: 15 May 2015 /Published online: 27 May 2015

(C) Springer Science+Business Media New York 2015

\begin{abstract}
This review evaluated a total of 22 research papers on observational learning as it applies to individuals diagnosed with autism spectrum disorders. The researchers divided the papers into three domains. The first domain was comparing the observational learning behavior of individuals diagnosed with autism spectrum disorders to typically developing and non-typically developing individuals. The second domain was how observational learning was taught to individuals diagnosed with autism spectrum disorders. The third domain was the reported levels of observational learning as an ancillary measure. The results of this review have both implications for clinicians as well as future areas of research which should be investigated.
\end{abstract}

Keywords Autism · Generalized imitation · Imitation · Observational learning

Research has indicated that humans are capable of learning simply by observing others come into contact with responsestimulus contingencies (Catania, 2007). According to Catania

Justin B. Leaf

jblautpar@aol.com

Donna Townley-Cochran

donnalynntc@gmail.com

Mitchell Taubman

mtautpar@aol.com

Ronald Leaf

Rlautpar@aol.com

John McEachin

Jmautpar@aol.com

1 Autism Partnership Foundation, 200 Marina Drive Seal, Beach, CA 90740, USA
(2007), one must demonstrate a variety of prerequisite behaviors in order to successfully acquire skills through observation. These behaviors include, but are not limited to, generalized imitation, self-awareness, verbal behavior, and the ability to discriminate behavior (Catania, 2007). However, individuals with autism demonstrate delays across these prerequisite skill sets (Lovaas 2003), which could explain why individuals with autism may not learn through observation as readily as peers that are typically developing.

The significance of observational learning for children who are typically developing has been widely investigated. In a seminal study on observational learning, Bandura et al. (1961) found that children who are typically developing imitated an adult model after observing the adult engage in a discrimination task. The children not only imitated the model's correct response, but the "extra" responses the model made while engaging in the correct response. For example, if the adult knocked over a doll in order to complete the task, the child knocked over the doll. If the adult set the doll on the ground in order to complete the task, the child set the doll on the ground. In a later study, Bandura, Ross, and Ross (1963) further evaluated observational learning by evaluating the behavior toward a bobo doll of children who are typically developing following an observational period of a peer's interaction with the same doll. In this study, the typically developing peer aggressed toward the doll; half of the children observed the model receive treats following the aggressive behavior toward the doll and the other half observed the model receive harassment from another peer following their aggressive behavior toward the doll. The results indicated that the children who observed the rewarding consequences were more likely to engage in aggressive behaviors than the children who observed the punitive consequences following the aggression. In a similar investigation, Bandura (1965) demonstrated that children not only had to observe the modeling 
response, but had to also observe the consequence for the modeling response for imitation to occur.

More recently, in an effort to streamline the definition of observational learning for future investigators within the context of learning and skill acquisition, Greer, Singer-Dudek, and Gautreaux (2006) defined observational learning as the process of acquiring a skill, or set of skills, as a result of observing others come in contact with contingencies of reinforcement or punishment. Throughout the remainder of this paper, observational learning will be referred to as the observation of others coming in contact with consequences and the subsequent acquisition of new responses or conditioning of reinforcers as a result of these observations.

When it comes to acquiring new skills, children who are typically developing demonstrate high rates of learning, as well as rapid expansion of a core repertoire that can serve as a foundation for the occurrence of observational learning. For individuals with autism, however, intervention typically involves teaching skills in a oneto-one instructional format (Smith 2001). As a result, opportunities for learning through observation are significantly reduced, subsequently limiting the number of learning opportunities the individual is exposed to. Furthermore, specific and sometimes extensive programmatic efforts are required to ensure that skills taught in one-to-one instruction will generalize to naturally occurring settings (Stokes and Baer 1977). Even when instructors employ techniques to foster generalization, many times individuals with autism only acquire a few skills over the course of several days or even weeks of instruction. For example, Williams, Carnerero, and Perez-Gonzales (2006) taught children to generalize labeling of two different actions, sleeping and playing ball, in the presence of a live model. For sleeping, the three participants required 63,25 , and 32 trials to reach criterion-level responding. For playing ball, two participants required 105 trials, and one required 42 trials to reach criterion-level responding. In an analysis of learning curves, Erdodi, Lajiness-O'Neill, and Schmitt (2013) discovered that individuals with autism, in comparison to peers who are typically developing and children with other disabilities, did not perform as well on a visual learning task requiring the participant to point to the same dots on a card as was previously demonstrated by the instructor. In fact, the authors suggest that individuals with autism may not benefit merely from exposure to novel or complex visual stimuli. In an effort to address this skill deficit, it would be a tremendous disadvantage to require formal one-to-one instructional formats and extensive instruction for the majority of new learning. Many facilities do not have the financial resources to accommodate one-to-one direct instruction
(Plavnick and Hume 2014). Therefore, it is highly recommended that clinicians identify and employ strategies to ensure observational learning will occur (Leaf and McEachin 1999; Lovaas 1981).

In an effort to provide clinicians with strategies to increase observational learning skills in individuals with autism, some curriculum guides offer support (Leaf and McEachin 1999; Lovaas 1981; 2003; Maurice et al. 2001; Taubman et al. 2011). For example, in one of the earliest curriculum books for individuals with autism, Lovaas (1981) provides six activities to practice and acquire skills that make observational learning more likely to occur. Leaf and McEachin (1999) also provide goals of observational learning and a sequence of phases to build skills that could lead to individuals increasing their ability to learn through observation. Other curriculum guides do not explicitly discuss observational learning, but provide programs that target skills that have been identified as foundational skills (Taubman et al. 2011; Taylor and DeQuinzio 2012) that allow individuals to learn through observation. These skills include building a generalized imitative repertoire (including imitating peers) (Lovaas 2003; Maurice et al. 2001) and discrimination learning (Lovaas 2003).

In addition to clinicians' work with observational learning, over the last several decades, various reviews have identified that there has been a growing body of empirical literature with respect to observational learning and individuals with autism (Plavnick and Hume 2014; Robertson and Biederman 1989; Taylor and DeQuinzio 2012).

Some researchers have conducted reviews exploring the literature of observational learning with individuals with autism (Plavnick and Hume 2014; Robertson and Biederman 1989; Taylor and DeQuinzio 2012). Robertson and Biederman (1989) recommended that more research should be conducted using valid experimental designs. Taylor and DeQuinzio (2012) outlined the foundational skills required for individuals to learn through observation, as well as suggestions for ways to increase the probability that observational learning would occur in a teaching environment. Most recently, Plavnick and Hume (2014) offered a review of a sample of literature covering observational learning with individuals with autism. The authors recommended that a systematic review of the literature be conducted. To determine how the research literature has addressed these areas of future direction, as well as identify additional areas, a systematic review of the literature is in fact necessary. The purpose of this review is to systematically review and identify the existing literature with respect to observational learning to provide both researchers and clinicians with areas that require future focus for individuals with autism. 


\section{Method}

\section{Article Selection}

In order to identify articles pertaining to observational learning for individuals with autism, researchers conducted two types of searches through electronic sources: a general search utilizing PsycInfo and a focused search utilizing eight peerreviewed journals. These eight journals were selected based on their reputation of publishing studies of topics involving individuals with autism. Table 1 lists the specific information researchers used for article selection including the electronic source, the web address at which the source can be found, the years searched, the search terms used, the number of search results, and number of articles selected for further review from each electronic source. Specific years searched and search terms varied depending on the electronic source, but were restricted to including articles published between the years 1960 and 2013. Search terms included observational learning and autism, with the exception of one journal (behavior analysis in practice) in which the researcher conducted a manual electronic search through archives. The number of search results varied depending on the source, ranging from 0 to 297. In order to be selected for review, the study obtained from the search results had to include two components in the title, keywords, or abstract. These two components were (1) inclusion of a term, phrase or description of observational learning, and (2) indication that the study was targeted for individuals with autism. Terms also accepted when describing the population included autistic, autistic-like, pervasive developmental disorder (PDD), or developmental disabilities; terms such as mental retardation and intellectual disabilities were excluded from review. A total of 30 articles from the general search and 6 from the focused search met this criterion and were further reviewed.

\section{Article Review}

The first step of the review process was to identify if the study would be applicable to the autism population, as well as that they collected and provided measurable data. As such, the researchers reviewed each article to determine that at least one individual with autism (described as autistic, autisticlike, or PDD) participated in the study. In addition, the study was reviewed to determine if it reported direct measures for the skills in which learning through observation could have occurred. If the study included both components, the researchers continued to review the article. If the study did not have any participants with autism, or did not provide direct measures of skills in which learning through observation could have occurred, that article was excluded from further review and coding. After this portion of the review, 24 out of the 36 articles selected met these criteria.
The next step of the review process involved identifying if participants were able to learn through observation in an in vivo situation. Articles that utilized video modeling (unless the article also included in vivo opportunities) were excluded. Articles that allowed in vivo opportunities for learning through observation were further reviewed. Articles that did not utilize in vivo opportunities were excluded from subsequent coding. After this portion of the review, 18 of the 24 articles met criteria.

While reviewing 36 the articles, 2 additional articles frequently referenced were identified that met criteria used during the review process (Charlop et al. 1983; Coleman and Stedman 1974). With the addition of these 2 articles, a total of 20 articles met review criteria and proceeded to the coding process.

\section{Coding}

During the coding process, researchers read each article to determine the number of participants and age range, history of imitation and learning through observation, skill(s) measured, how observational learning targets were taught, characteristics of the model, when the skill was probed, and the outcome of each article.

Number of Participants and Age Range The number of participants was defined as the number of individuals the article reported with autism, PDD or described as autistic or autistic-like. Participants within the same study without this description (e.g., participants diagnosed with mild mental retardation) were excluded from this count. In order to better understand the ages for which observational learning has been studied, researchers identified the age range of the participants.

History of Imitation and Learning Through Observation Researchers sought to determine if participants demonstrated pre-requisites or had a history of learning through observation, as this could influence responding. As such, information was collected to determine participants' ability to engage in imitation and/or learn through observation prior to the start of the study.

Skill(s) Measured To identify one aspect of generality of the skills potentially learned through observation, the skill(s) potentially acquired through observation were identified.

How Observational Learning Targets Were Taught The exposure to learning through observation can vary with respect to how the skills are acquired. Strategies can be used to teach a skill that would subsequently increase the likelihood that observational learning would occur; this was identified as direct instruction. Another way in which individuals can learn through observation is by indirect instruction. Studies 


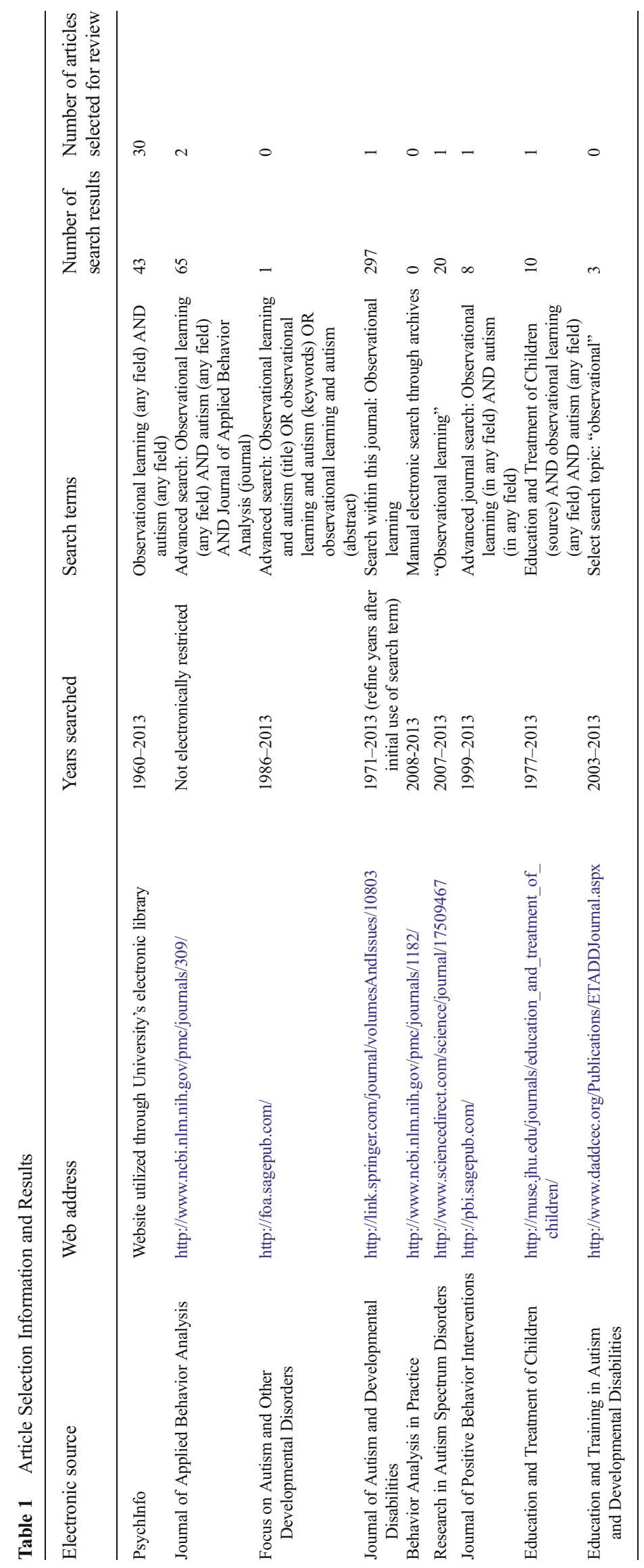


identified as teaching targets through indirect instruction set up an opportunity in which the participant observed a model come in contact with the relevant contingencies and an opportunity was subsequently provided for the participant to demonstrate the desired response.

Model and Format For each article, the type of model, adult or peer, was identified. To determine the specific teaching format, the type of teaching format was also identified. The teaching format was labeled one-to-one if there was an instructor, model, and participant present. The teaching format was labeled as group instruction if at least two models and the participant were present during opportunities to learn through observation.

When Observational Learning Targets Were Probed To determine how researchers measured progress with respect to the acquisition of skills exposed to opportunities to learn through observation, when probes were conducted was identified.

Outcome To assess the ability of individuals with autism to learn through observation, general results were identified for each article.

\section{Results}

In total, 20 articles were coded. Table 2 depicts the results of the coding process.

\section{Number of Participants and Age Range}

Across 20 articles, a total of 73 individuals with autism or PDD or described as autistic or autistic-like participated in a research study in which they were exposed to opportunities for learning through observation. The age range of these 73 individuals was 3 to 16 years.

\section{History of Imitation and Learning Through Observation}

Out of the 20 articles, only six reported that all participants engaged in imitative responding, while one study reported imitative responding for three of the four participants. This equals 46 individuals for which information was not provided to determine their ability to engage in imitative responding. Only one study (Pereira-Delgado and Greer 2009) reported that the participants did not previously learn through observation; no other study reported participants' history of learning through observation.

\section{Skill(s) Measured}

Overall, 21 different skills were targeted across all 20 studies. These skills included receptive labels (Charlop et al. 1983; Rehfeldt et al. 2003), expressive labels (Coleman and Stedman 1974; Leaf et al. 2013), voice volume (Coleman and Stedman 1974), play (Colozzi et al. 2008; Tyron and Keane 1986), various discriminations (Egel et al. 1981; Varni et al. 1979), imitation (Ganz et al. 2008), general knowledge skills (Ihrig and Wolchik 1988), conditioning preferences/ reinforcers (Leaf et al. 2012; Singer-Dudek and Oblak 2013), job functions (Leaf et al. 2013), inferences (Leaf et al. 2013), sight words (Ledford et al. 2008), monitoring response (Pereira-Delgado and Greer 2009; Taylor et al. 2012), reading (Rehfeldt et al. 2003), picture to word/word to picture matching (Rehfeldt et al. 2003), eating (Sira and Fryling 2012), food and drink prep (Tekin-Iftar and Birkan 2010), sign language (Venn et al. 1996), spelling (Werts et al. 1996), playing an audiotape (Werts et al. 1996), using a calculator (Werts et al. 1996), and social communication (Wilson 2013).

\section{How Observational Learning Targets Were Taught}

Three studies used direct instruction to set up opportunities to learn through observation (Ganz et al. 2008; Pereira-Delgado and Greer 2009; Taylor et al. 2012). Seventeen targeted observational learning indirectly (Charlop et al. 1983; Coleman and Stedman 1974; Colozzi et al. 2008; Egel et al. 1981; Ihrig and Wolchik 1988; Leaf et al. 2012, 2013; Ledford et al. 2008; Rehfeldt et al. 2003; Singer-Dudek and Oblak 2013; Sira and Fryling 2012; Tekin-Iftar and Birkan 2010; Tyron and Keane 1986; Varni et al. 1979); of those 17, only one specifically sought to compare learning through observation of individuals with autism compared to peers who are typically developing (Varni et al. 1979).

Across the three studies that used direct instruction, each study utilized a different procedure to teach skills to increase the likelihood that observational learning would occur. Through a series of prompting techniques, one study attempted to teach four participants with autism to imitate peers (Ganz et al. 2008). During each session, the participants sat together in a group. Out of the group of participants, the teacher identified a "leader," placing a necklace around this person. The leader remained the same throughout the entire 4min session, and was rotated over the following days to allow each participant an opportunity to be the leader. The leader was provided with several items for playing. The remaining participants were not instructed to imitate the peer, but the text "do what the leader is doing," was placed on the corner of the participants' desks. If the children did not imitate the leader, the trainer progressed through the following prompting levels: (1) when the next interval started, the teacher gave the 


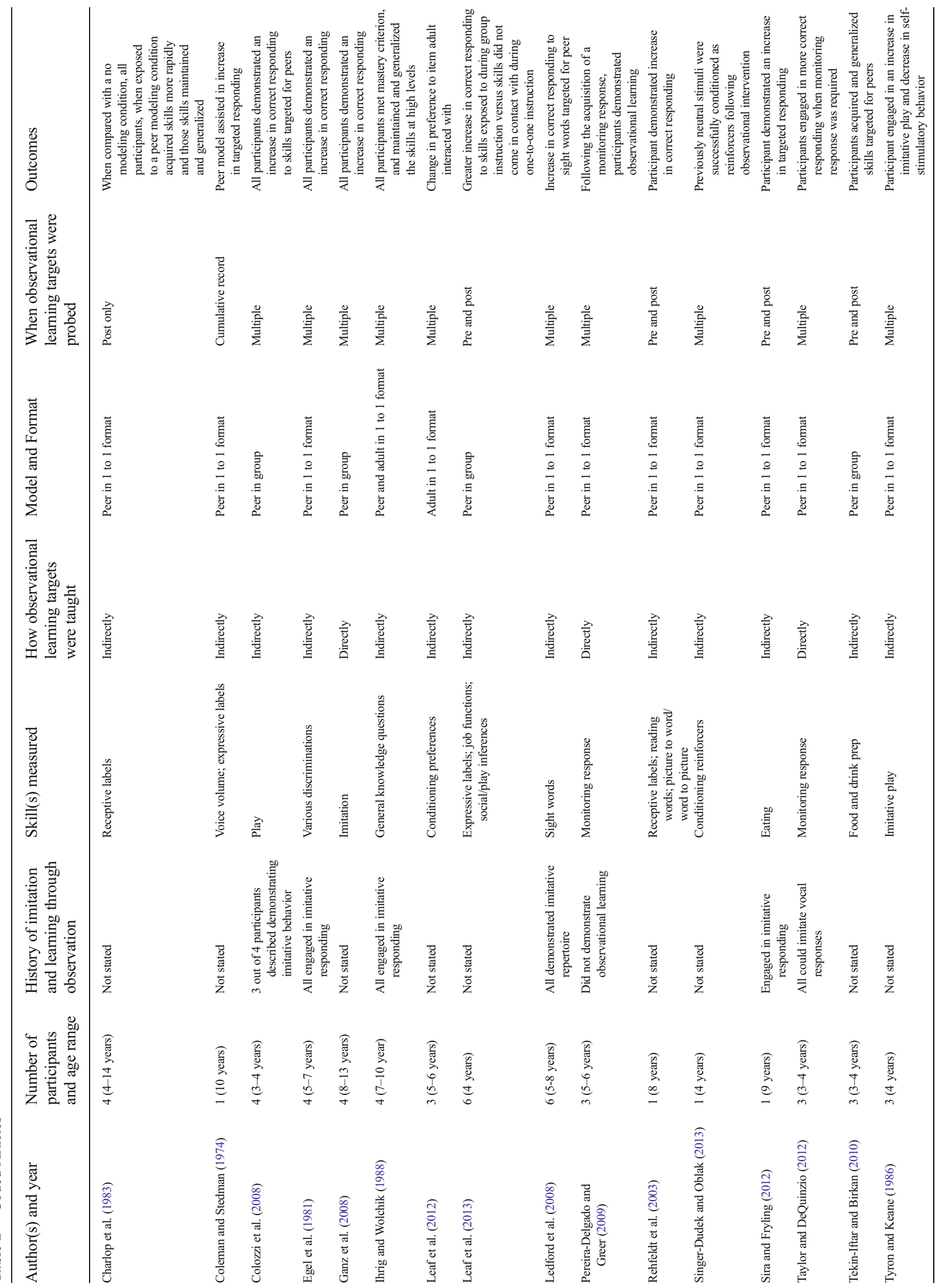




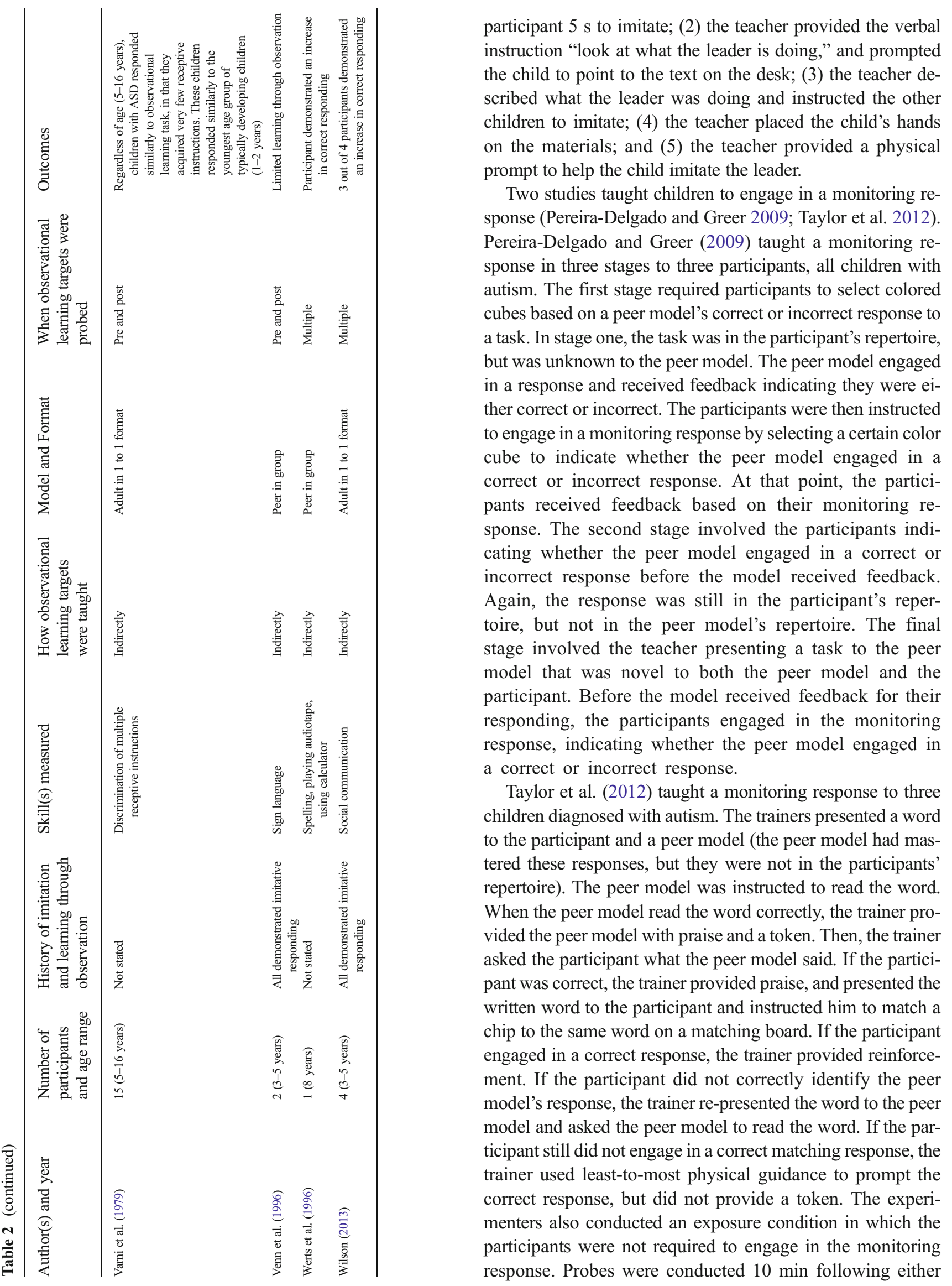


training session or exposure session. During probe sessions, participants did not receive reinforcement or prompts.

\section{Model and Format}

A majority of the studies, 16 (80\%), utilized peer models (Charlop et al. 1983; Coleman and Stedman 1974; Colozzi et al. 2008; Egel et al. 1981; Ganz et al. 2008; Leaf et al. 2013; Ledford et al. 2008; Pereira-Delgado and Greer 2009; Rehfeldt et al. 2003; Singer-Dudek and Oblak 2013; Sira and Fryling 2012; Taylor et al. 2012; Tekin-Iftar and Birkan 2010; Tyron and Keane 1986; Venn et al. 1996; Werts et al. 1996), while 3 studies utilized adult models (Leaf et al. 2012; Varni et al. 1979; Wilson 2013). One study utilized both adult and peer models in an effort to compare the effectiveness of the two different types of models (Ihrig and Wolchik 1988). In addition, 14 of the 20 studies set up opportunities to learn through observation in a one-to-one teaching format (Charlop et al. 1983; Coleman and Stedman 1974; Egel et al. 1981; Ganz et al. 2008; Ihrig and Wolchik 1988; Leaf et al. 2012; Ledford et al. 2008; Pereira-Delgado and Greer 2009; Rehfeldt et al. 2003; Singer-Dudek and Oblak 2013; Sira and Fryling 2012; Taylor et al. 2012; Tyron and Keane 1986; Varni et al. 1979; Wilson 2013) versus six studies that instead used a group-teaching format (Colozzi et al. 2008; Leaf et al. 2013; Tekin-Iftar and Birkan 2010; Venn et al. 1996; Werts et al. 1996).

\section{How Observational Learning Targets Were Probed}

Out of the 20 articles, only 12 measured skill acquisition multiple times throughout baseline, intervention, and maintenance or generalization if applicable (Colozzi et al. 2008; Egel et al. 1981; Ganz et al. 2008; Ihrig and Wolchik 1988; Leaf et al. 2012; Ledford et al. 2008; Pereira-Delgado and Greer 2009; Singer-Dudek and Oblak 2013; Taylor et al. 2012; Tyron and Keane 1986; Werts et al. 1996; Wilson 2013). Six studies determined progress utilizing only pre and post probes (Leaf et al. 2013; Rehfeldt et al. 2003; Sira and Fryling 2012; TekinIftar and Birkan 2010; Varni et al. 1979; Venn et al. 1996). In one study, while pretests were included in the text of the article, the researchers only reported data for post probe responding (Charlop et al. 1983). Lastly, one study utilized a cumulative record to measure progress (Coleman and Stedman 1974).

\section{Outcome}

One study identified that when compared to peers that are typically developing, individuals with autism acquire less responses when presented with an opportunity to learn through observation. However, a majority of the studies reported optimistic results, demonstrating that children with autism have the capability of learning through observation. Three studies, through direct instruction, taught skills to ensure observational learning would occur. Of the remaining 17 articles that utilized indirect instruction, one study found that individuals with autism switched preferences (Leaf et al. 2012), another found that previously neutral stimuli could be conditioned as reinforcers (Singer-Dudek and Oblak (2013)), and 14 studies reported either the occurrence of learning through observation (Charlop et al. 1983; Varni et al. 1979) or an increase in correct responding (Coleman and Stedman 1974; Colozzi et al. 2008; Egel et al. 1981; Ihrig and Wolchik 1988; Leaf et al. 2013; Ledford et al. 2008; Rehfeldt et al. 2003; Sira and Fryling 2012; Tekin-Iftar and Birkan 2010; Tyron and Keane 1986; Werts et al. 1996; Wilson 2013). Only one study (Venn et al. 1996) identified that limited learning through observation occurred.

\section{Discussion}

The purpose of this review was to first evaluate the existing literature on observational learning with individuals with autism to provide recommendations for future directions for both researchers and clinicians. Over a span of 54 years, researchers were only able to identify a limited number of studies that provided opportunities for learning through observation for individuals with autism. The results of the search further confirm that learning through observation is a significant area of deficit for individuals with autism. The results additionally indicate that a few number of studies specifically target teaching a skill to ensure observational learning. As such, behavioral interventions should focus on systematically teaching skills that will ensure observational learning.

Individuals with autism lack the skills necessary to benefit from observational learning (Varni et al. 1979), which highlights the importance of teaching skills to increase the likelihood of observational learning occurring (Plavnick and Hume 2014; Robertson and Biederman 1989; Taylor et al. 2012). Currently, there are explicit procedures and strategies to teach skills to foster learning through observation for individuals with autism in curriculum books (Leaf and McEachin 1999; Lovaas 1981). Given these two pieces of information, one would have expected that there would be numerous studies on teaching the skills necessary for individuals with autism to learn through observation. However, our review only found three studies where skills were directly targeted to increase observational learning. These three studies had only 4,3 , and 3 participants, respectively, and each study utilized different procedures. The outcomes of each study suggested that a systematic intervention can allow individuals with autism to learn through observation (Ganz et al. 2008; Pereira-Delgado and Greer 2009; Taylor and DeQuinzio 2012). 
Of the 20 studies reviewed in this paper, 17 (85\%) reported the indirect instruction of skills acquired through observation. Across these 17 studies, a variety of observational tasks were measured, a majority of studies utilized peer models, and teaching was conducted primarily in one-to-one teaching formats. Pre and post or multiple probe measures were reported for 15 of the 17 studies. Overall, this field of research tends to show that learning through observation can occur for individuals with autism. However, these 17 studies may leave us with more questions than answers. For one, 10 of the 17 studies $(59 \%)$ do not report participants' history of imitative responding or learning through observation; it is not known if the participants had generalized imitation prior to intervention. Second, none of the studies stated whether the participants had a previous history of training that provided opportunities for learning through observation. It is possible that the participants had a previous history of training within a teaching format that provided opportunities for learning through observation, which may have led to the higher rates of correct responding; if the participants did not have a previous history with learning through observation, then these results bring up the question of whether direct instruction is necessary for observational learning to occur.

\section{Recommendations}

Based upon the findings in this review, general recommendations for both research and for clinical practice are provided.

Research First, it is clear that learning through observation is a deficit for individuals with autism; however, there are few studies that have evaluated teaching skills to increase the likelihood that observational learning will occur. More research needs to be conducted on methods to teach skills to individuals with autism that would increase the likelihood that observational learning would occur. Researchers can start by evaluating the procedures described in curriculum books (Leaf and McEachin 1999; Lovaas 1981) or by creating their own procedures to identify additional techniques to teach skills to foster observational learning in individuals with autism. Researchers must evaluate the procedure across a wide variety of participants (e.g., variety of ages, functioning levels, gender) and across a wide variety of skills (e.g., receptive identification, labeling, non-verbal imitation, play). Future researchers should also evaluate teaching skills to ensure observational learning will occur in different instructional formats (e.g., one-to-one versus group instructional formats) and in different settings (e.g., university, home, clinic, school, and community). To provide stronger empirical evidence, researchers should evaluate participants' skill levels both prior to and following intervention in order to provide sufficient evidence of effective interventions. If further research confirms that learning through observation can be taught successfully, then researchers should proceed to compare the different methods of teaching to identify not only the most effective, but the most efficient methods.

Second, there needs to be more studies comparing the rate at which individuals learn through observation across several different parameters. Researchers should continue to compare individuals with autism to other populations to assess each population's ability to learn through observation; to date, only one study has done so (Varni et al. 1979). Future researchers should also compare the rate of learning through observation on new tasks for individuals who have been explicitly taught skills to promote observational learning to those who have not been explicitly taught those skills. This area of research would require researchers to more thoroughly describe their participants' history of learning through observation when publishing effective interventions. Researchers should also conduct evaluations in a more systematic manner to assess how the acquisition of proposed prerequisite responses (Taylor and DeQuinzio 2012) affects the development of learning through observation. In doing so, this would make it easier for researchers to identify and describe relevant and meaningful prerequisite skills when outlining participants' history of learning through observation.

Finally, future researchers should evaluate whether learning through observation truly does produce higher rates of overall learning. It is presumed that when an individual is able to learn through observation, their overall rate of learning will increase; however, this has not been proven for individuals with autism. Future researchers could compare a group of children who have received training on target skills to increase the likelihood of observational learning to a group of children who have not received training and evaluate whether overall rates of learning (e.g., language development, social development, and academic development) are higher for the children who have received explicit training. The researchers could simultaneously track which skills are directly targeted to the child and which are not directly targeted, as this would show which skills are learned along the way without explicit training.

Clinical Recommendations While the above recommendations have been geared toward researchers, it is important that clinicians understand that they play a key role in the development of the applied literature in this field. Taylor and Dequinzio (2012) recommend that clinicians provide children with as many opportunities as possible for learning through observation to occur. Because research investigations have not kept pace with development of clinical interventions, it makes sense for clinicians to pursue or continue to pursue their efforts to expand teaching skills to ensure observational learning will occur and not wait for the research to catch up to the clinical practice. Clinicians may wish to start with presenting activities suggested within various curriculum guides that outline the development of skills that increase the likelihood that 
observational learning will occur (Lovaas 1981; Leaf and McEachin 1999). Overtime, clinicians should modify and adapt these procedures to target specific individuals. When this does occur, it is crucial for clinicians and researchers to indicate how the techniques derived from various curriculum guides influenced their current intervention technique. Clinicians should be encouraged to report their findings as case studies in empirical journals to help disseminate their efforts and help other consumers understand the procedures, as well as aid researchers in formulating more specific and productive research hypotheses. When clinicians are able to produce gains in observational learning, they have the ability to promote future applied research.

\section{Conclusion}

In conclusion, this review systematically evaluated the emerging observational learning literature with individuals with autism. The current body of literature provides individuals with autism the opportunity to learn through observation, provides techniques for individuals with autism to engage in skills to ensure observational learning will occur, and one study compares individuals with autism to individuals that are typically developing. While individuals with autism demonstrate deficits within this area, there was only one study that compared individuals with autism to other populations. In addition, there are a limited number of studies that have actually taught skills to guarantee observational learning will occur. If the goal of intervention for individuals is to not only treat areas of deficit, but to establish a rate of learning that is similar to typically developing peers for certain individuals, learning through observation is a vital for these individuals. Based on the findings of this review, several recommendations were made for researchers to extend the observational learning literature, as well as for clinicians to pursue or continue to pursue teaching skills to ensure observational learning will occur, and facilitating opportunities for individuals to learn through observation. The collaborative effort between researchers and clinicians should spark future growth and development within the literature to promote new and empirically based strategies to allow individuals with autism to learn through observation.

Conflict of Interest The authors have no declared conflict of interests with the manuscript entitled "Observational Learning for Students Diagnosed with Autism: A Review Paper.”

\section{References}

Bandura, A. (1965). Influence of models' reinforcement contingencies on the acquisition of imitative responses. Journal of Personality and Social Psychology, 1, 589-595.
Bandura, A., Ross, D., \& Ross, S. A. (1961). Transmission of aggression through imitation of aggressive models. Journal of Abnormal and Social Psychology, 63, 575-582.

Bandura, A., Ross, D., \& Ross, S. A. (1963). Vicarious reinforcement and imitative learning. Journal of Abnormal and Social Psychology, 67, 601-607.

Charlop, M. H., Schreibman, L., \& Tryon, A. S. (1983). Learning through observation: the effects of peer modeling on acquisition and generalization in autistic children. Journal of Abnormal Child Psychology, 11(3), 355-366. doi:10.1007/BF00914244.

Coleman, S. L., \& Stedman, J. M. (1974). Use of a peer model in language training in an echolalic child. Journal of Behavior Therapy and Experimental Psychology, 5, 275-279.

Colozzi, G. A., Ward, L. W., \& Crotty, K. W. (2008). Comparison of simultaneous prompting procedure in 1:1 and small group instruction to teach play skills to preschool students with pervasive developmental disorder and developmental disabilities. Education and Training in Developmental Disabilities, 43(2), 226-248.

Egel, A. L., Richman, G. S., \& Koegel, R. L. (1981). Normal peer models and autistic children's learning. Journal of Applied Behavior Analysis, 14(1), 3-12. doi:10.1901/jaba.1981.14-345.

Erdodi, L., Lajiness-O’Neill, R., \& Schmitt, T. A. (2013). Learning curve analyses in neurodevelopmental disorders: are children with autism spectrum truly visual learners? Journal of Autism and Developmental Disorders, 43, 880-890.

Ganz, J. B., Bourgeois, B. C., Flores, M. M., \& Campos, B. A. (2008). Implementing visually cued imitation training with children with autism spectrum disorders and developmental delays. Journal of Positive Behavior Interventions, 10, 56-66. doi:10.1177/ 1098300707311388.

Greer, R. D., Singer-Dudek, J., \& Gautreaux, G. (2006). Observational learning. International Journal of Psychology, 41, 486-499.

Ihrig, K., \& Wolchik, S. A. (1988). Peer versus adult models and autistic children's learning: acquisition, generalization, and maintenance. Journal of Autism and Developmental Disorders, 18(1), 67-79. doi:10.1007/BF02211819.

Leaf, R., \& McEachin, J. (1999). A work in progress. New York: DRL.

Leaf, J. B., Oppenheim-Leaf, M. L., Leaf, R., Courtemanche, A. B., Taubman, M., McEachin, J., Sheldon, J. B., \& Sherman, J. A. (2012). Observational effects on the preferences of children with autism. Journal of Applied Behavior Analysis, 45(3), 473-483. doi:10.1901/jaba.2012.45-473.

Leaf, J. B., Tsuji, K. H., Lentell, A. E., Dale, S., Kassardjian, A., Taubman, M., McEachin, J., Leaf, R., \& Oppenheim-Leaf, M. L. (2013). A comparison of discrete trial teaching implemented in a one-to-one instructional format and in a group instructional format. Behavioral Interventions, 28(1), 82-106. doi:10.1002/bin.1357.

Ledford, J. R., Gast, D. L., Luscre, D., \& Ayres, K. M. (2008). Observational and incidental learning by children with autism during small group instruction. Journal of Autism and Developmental Disorders, 38, 86-103. doi:10.1007/s10803-007-0363-7.

Lovaas, I. O. (1981). Expanding your child's world: observational learning. In I. O. Lovass (Ed.), Teaching developmentally disabled children: the me book (pp. 203-207). Baltimore, Maryland: University Park Press.

Lovass, I. O. (2003). Teaching individuals with developmental disabilities. Austin: Pro Ed.

Maurice, C., Green, G., \& Foxx, R. M. (2001). Making a difference: behavioral interventions for autism. Austin: Pro Ed.

Pereira-Delgado, J., \& Greer, R. D. (2009). The effects of peer monitoring training on the emergence of the capability to learn from observing instruction received by peers. The Psychological Record, 59, 407-434.

Plavnick, J. B., \& Hume, K. A. (2014). Observational learning by individuals with autism: a review of teaching strategies. Autism, 18, 458-466. 
Rehfeldt, R. A., Latimore, D., \& Stromer, R. (2003). Observational learning and the formation of classes of reading skills by individuals with autism and other developmental disabilities. Research in Developmental Disabilities, 24(5), 333-358. doi:10.1016/S08914222(03)00059-3.

Robertson, H. A., \& Biederman, G. B. (1989). Modeling, imitation, and observational learning in remediation experimentation 1979-1988: an analysis of the validity of research designs and outcomes. Canadian Journal of Behavioral Science, 21, 174-197.

Singer-Dudek, J., \& Oblak, M. (2013). Peer presence and the emergence of conditioned reinforcement from observation. Journal of Applied Behavior Analysis, 46(3), 592-602. doi:10.1901/jaba.2013.46-592.

Sira, B. K., \& Fryling, M. J. (2012). Using peer modeling and differential reinforcement in the treatment of food selectivity. Education and Treatment of Children, 35(1), 91-100.

Smith, T. (2001). Discrete trial training in the treatment of autism. Focus on Autism and Other Developmental Disabilities, 16, 86-92.

Stokes, T. F., \& Baer, D. M. (1977). An implicit technology of generalization. Journal of Applied Behavior Analysis, 10(2), 349-367.

Taubman, M., Leaf, R., \& McEachin, J. (2011). Crafting connections: contemporary applied behavior analysis for enriching the social lives of persons with autism spectrum disorder. New York: DRL.

Taylor, B., \& DeQuinzio, J. A. (2012). Observational learning and children with autism. Behavior Modification, 36, 341-360.

Taylor, B. A., DeQuinzio, J. A., \& Stine, J. (2012). Increasing observational learning of children with autism: a preliminary analysis. Journal of Applied Behavior Analysis, 45(4), 815-820. doi:10. 1901/jaba.2012.45-815.
Tekin-Iftar, E., \& Birkan, B. (2010). Small group instruction for students with autism: general case training and observational learning. Journal of Special Education, 44(1), 50-63. doi:10.1177/ 0022466908325219.

Tyron, A. S., \& Keane, S. P. (1986). Promoting imitative play through generalized observational learning in autistic like children. Journal of Abnormal Child Psychology, 14(4), 537-549. doi:10.1007/ BF01260522.

Varni, J. W., Lovaas, O. I., Koegel, R. L., \& Everett, N. L. (1979). An analysis of observational learning in autistic and normal children. Journal of Abnormal Child Psychology, 7(1), 31-43. doi:10.1007/ BF00924508.

Venn, M. L., Wolery, M., \& Greco, M. (1996). Effects of every-day and every-other-day instruction. Focus on Autism and Other Developmental Disabilities, 11(1), 15-28. doi:10.1177/ 108835769601100103.

Werts, M. G., Caldwell, N. K., \& Wolery, M. (1996). Peer modeling of response chains: observational learning by students with disabilities. Journal of Applied Behavior Analysis, 29(1), 53-66. doi:10.1901/ jaba.1996.29-53.

Williams, G., Carnerero, J. J., \& Perez-Gonzalez, L. A. (2006). Generalization of tacting actions in children with autism. Journal of Applied Behavior Analysis, 39, 233-237.

Wilson, K. P. (2013). Teaching social-communication skills to preschoolers with autism: efficacy of video versus in vivo modeling in the classroom. Journal of Autism and Developmental Disorders, 43, 1819-1831. 